\title{
Metastable exotic multihypernuclear objects
}

\author{
Jürgen Schaffner \\ Institut für Theoretische Physik, Johann Wolfgang Goethe Universität, Frankfurt, Germany \\ Carsten Greiner \\ Institut für Theoretische Physik, Universität Erlangen-Nürnberg, Erlangen, Germany \\ Horst Stöcker \\ Institut für Theoretische Physik, Johann Wolfgang Goethe Universität, Frankfurt, Germany
}

(Received 10 December 1991)

\begin{abstract}
Relativistic heavy ion collisions constitute a prolific source of hyperons: tens of hyperons per event are predicted at energies $E \geq 10 \mathrm{GeV} /$ nucleon, providing a scenario for the formation of metastable exotic multihypernuclear objects. They may exhibit exceptional properties: bound neutral (e.g., ${ }^{4} M_{2 \Lambda}^{2 n}$, ${ }^{10} M_{2 \Lambda}^{8 n}$, pure $\Lambda$ droplets, ${ }^{8} \Lambda$ ) and even negatively charged composites objects with positive baryon number (e.g., ${ }^{4} M_{2 \Sigma^{-}}^{2 n},{ }^{6} M_{2 \Lambda 2 \Xi^{-}}^{2 n}$ ) could be formed in rare events. Such negative nuclei can easily be identified in a magnetic spectrometer. They could be considerably more abundant than antinuclei of the same $A$. We use the relativistic meson-baryon field theory-which gives an excellent description of normal nuclear and single- $\Lambda$ hypernuclear properties - to calculate the rich spectrum of such exotic objects, their stability, and their structure. We also find solutions for a large variety of bound short-lived nuclei (e.g., ${ }^{8} M_{2 \Lambda, 2 \Sigma^{-}}^{2 p 2 n}$ ), which may decay strongly via formation of cascade ( $\Xi$ ) particles. Multi- $\Xi$ hypernuclei are also evaluated. A variety of potential candidates for such metastable exotic nuclei is presented. It turns out that the properties of such exotic multihypernuclear objects reveal quite similar features as the strangelet proposed as a unique signature for quark-gluon plasma formation in heavy ion collisions.
\end{abstract}

PACS number(s): 25.75. $+\mathrm{r}, 21.80 .+\mathrm{a}$

\section{INTRODUCTION}

Hypernuclei allow the study of the effects of strangeness in nuclei (for an excellent recent review, see [1]). The experimental situation is as follows: The singleparticle energy of the lowest state of $\Lambda$ hypernuclei was measured from mass $A=3$ to 5 in early emulsion experiments [2]. There are also two older emulsion events of double- $\Lambda$ hypernuclei which are of interest for studying the $\Lambda-\Lambda$ interaction [3]. One of these emulsion events has been reanalyzed in [4]. Recently, a sequential weak decay of a double hypernucleus was found [5] and interpreted as a weak decay of ${ }^{13} B_{\Lambda \Lambda}$ [6]. Experiments at Brookhaven Laboratory using the reaction $\left(\pi^{+}, K^{+}\right)$[7] make it possible to study even deep-lying $\Lambda$-hypernuclear levels over a wide range of $A$. There are two well-established features of $\Lambda$ hypernuclei measured: The potential is about $30 \pm 3 \mathrm{MeV}$, and the spin-orbit splitting is lower than the experimental resolution [1]. On the contrary, the experimental situation on $\Sigma$ hypernuclei seems to be still controversial because of low statistics data [8]. One of the looked after problems concerns the unexpected existence of narrow $\Sigma$-hypernuclear levels [9]. A shoulder has been observed in the stopped $\left(K^{-}, \pi^{-}\right)$reaction on ${ }^{4} \mathrm{He}$ corresponding to bound $\Sigma$ 's [10]. Finally, we would like to mention that there also exist seven emulsion events of $\Xi$ hypernuclei, which are examined in Ref. [11].

The production of (multi)hypernuclei [12] in heavy-ion collisions has attracted much attention recently [13].
Cross sections for single- and double- $\Lambda$ hypernuclei have been estimated in [14] and have recently been measured in [15]. The properties of multihypernuclei $[13,16]$ are still one of the least-explored, open questions in hypernuclear research.

A (meta)stable state of matter containing abundant strangeness on the quark level (strangelets) would be a fascinating alternative $[16,17]$. A mechanism for strangelet formation in relativistic heavy-ion collisions based on a strong first-order phase transition has been proposed $[18,19]$ and recently been confirmed theoretically, although with mutually contradictory conclusions [20].

In this paper we want to investigate first the properties of hypernuclei and multihypernuclei, and second we extrapolate to the more exotic objects such as metastable $\Lambda$ droplets, neutron-hyperon droplets, and objects consisting of $\Lambda$ 's and $\Sigma$ 's, the lightest hyperons, with nucleons. This is done in a relativistic mean-field model (RMFM), which has been proven to give a very good description of normal nuclei [21-23] and $\Lambda$ hypernuclei $[13,24,25]$. We will compare both the metastable exotic multihypernuclear object (MEMO) and the strangelet pictures in the last section.

\section{SINGLE- $\Lambda$ HYPERNUCLEI IN THE RMFM}

We start from the Lagrangian given in [23]:

$$
\mathcal{L}=\mathcal{L}_{\text {Dirac }}+\mathcal{L}_{\phi}+\mathcal{L}_{V}+\mathcal{L}_{R}+\mathcal{L}_{A},
$$


TABLE I. Values used for the nucleonic coupling constants (taken from [23]).

\begin{tabular}{cccccc}
\hline \hline$g_{s N}$ & $g_{v N}$ & $g_{\rho N}$ & $m_{\sigma}(\mathrm{MeV})$ & $b\left(\mathrm{fm}^{-1}\right)$ & $c$ \\
\hline 7.51141 & 9.51541 & 4.92551 & 454.871 & -9.57568 & +1.97056 \\
\hline \hline
\end{tabular}

with additional terms for hyperons $\Lambda, \Sigma$, and $\Xi$ :

$$
\begin{aligned}
\mathcal{L}_{\text {Dirac }}^{\text {hyp }}= & \bar{\psi}_{\Lambda}\left(i \gamma^{v} \partial_{v}-m_{\Lambda}\right) \psi_{\Lambda}+\bar{\psi}_{\Sigma}\left(i \gamma^{v} \partial_{v}-m_{\Sigma}\right) \psi_{\Sigma} \\
& +\bar{\psi}_{\Xi}\left(i \gamma^{v} \partial_{v}-m_{\Xi}\right) \psi_{\Xi}, \\
\mathcal{L}_{\phi}^{\text {hyp }}=- & g_{\sigma \Lambda} \phi \bar{\psi}_{\Lambda} \psi_{\Lambda}-g_{\sigma \Sigma} \phi \bar{\psi}_{\Sigma} \psi_{\Sigma}-g_{\sigma \Xi} \phi \bar{\psi}_{\Xi} \psi_{\Xi}, \\
\mathcal{L}_{V}^{\text {hyp }=}- & g_{\omega \Lambda} V^{\mu} \bar{\psi}_{\Lambda} \gamma_{\mu} \psi_{\Lambda}-g_{\omega \Sigma} V^{\mu} \bar{\psi}_{\Sigma} \gamma_{\mu} \psi_{\Sigma} \\
& -g_{\omega \Xi} V^{\mu} \bar{\psi}_{\Xi} \gamma_{\mu} \psi_{\Xi}, \\
\mathcal{L}_{R}^{\text {hyp }=} & -\frac{1}{2} g_{\rho \Sigma} \mathbf{R}^{\mu} \cdot \bar{\psi}_{\Sigma} \tau \gamma_{\mu} \psi_{\Sigma}-\frac{1}{2} g_{\rho \Xi} \mathbf{R}^{\mu} \cdot \bar{\psi}_{\Xi} \tau \gamma_{\mu} \psi_{\Xi}, \\
\mathcal{L}_{\bar{A}}^{\text {hyp }=} & -\frac{1}{2} e A^{\mu} \bar{\psi}_{\Sigma}\left(1+\tau_{0}\right) \gamma_{\mu} \psi_{\Sigma}-\frac{1}{2} e A^{\mu} \bar{\psi}_{\Xi}\left(1+\tau_{0}\right) \gamma_{\mu} \psi_{\Xi} .
\end{aligned}
$$

Variation of the fields yields within the mean-field approach and the no-sea approximation the sum of the densities runs only over occupied states, four boson field and three baryon field equations, which can be solved numerically for the nuclear ground states [13].

The parameters of the meson fields and nucleon coupling are fitted to the properties of eight spherical nuclei. Calculations done with lower effective masses show instabilities for highly dense nuclear systems such as ${ }^{12} \mathrm{C}$ [26] and unphysical behavior in nuclear matter [27] for a negative coefficient $c$ in the standard nonlinear selfinteraction of the effective scalar field [28,29]. Note that for a smaller effective mass one always gets a negative coefficient $c$ (for a discussion of the parameter space and its shortcomings, see [30]). Recently, an extension of the standard model for arbitrary scalar self-interactions has been presented [31].

Here a fit with an effective mass of $m_{N}^{*} / m_{N}=0.75$ at $\rho_{0}$ [32] (see Table I) is chosen for the sake of stability. This is also consistent with the optical potential at low energies. It was shown in previous publications [13] that one can describe hypernuclear data also with lower

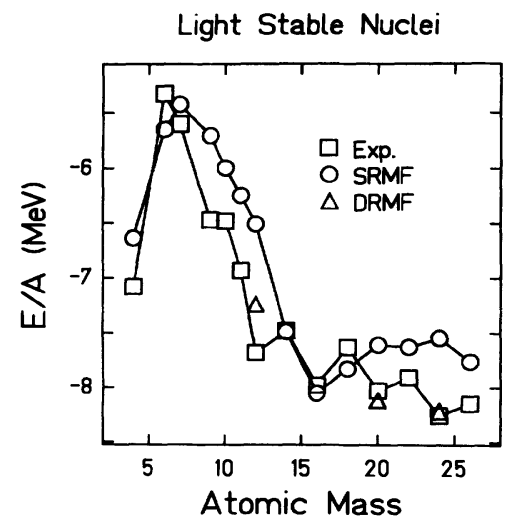

FIG. 1. Binding energy of light stable normal nuclei in the spherical relativistic mean-field model (SRMF) and in the deformed code (DRMF) compared with the experimental data. effective-mass parametrizations. The conclusion about hypernuclei is not affected by this smaller value of $m^{*}$ (see below).

This relativistic mean-field model has proven to give a good description of nuclear matter and finite normal nuclei $[21,23]$. Even for very light systems, one obtains surprisingly good results, as demonstrated in Fig. 1 . The two parameters for the $\Lambda$ couplings are adjusted to the recent $\left(\pi^{+}, K^{+}\right)$data from Brookhaven National Laboratory [7]. The coupling of the $\Sigma$ 's is assumed to be equal to the $\Lambda$ coupling, as suggested from the quark model picture [32,33] (hyperuniversal coupling). We consider two cases, varying either the two-hyperon coupling ratios $\alpha=g_{\sigma \Lambda} / g_{\sigma N}, \beta=g_{\omega \Lambda} / g_{\omega N}$ independently (full fit) or with the restriction that $\alpha=\beta$ (restricted fit). The two cases yield quite different coupling constants, namely, $\alpha=0.25$, $\beta=0.18$, and $\alpha=\beta=0.43$, respectively, which was also found for another parametrization with a lower effective mass [13]. Both sets reproduce the single-particle states well (Fig. 2 shows the restricted case). A small spin-orbit splitting comes out naturally because of the small coupling constants, which yield weak fields. This is an important advantage of the relativistic theory: The observed small spin-orbit splitting is predicted without requiring an additional fit parameter as, e.g., in the nonrelativistic case [23]. The binding energies of light hypernuclei from $A=5$ to 12 [2] agree also surprisingly well in our simple model (Fig. 3). The large difference in the couplings observed for the restricted and full fits can be understood when plotting the $\left(\chi^{2}\right)$ values in the plane of the two coupling ratios $\alpha=g_{\sigma \Lambda} / g_{\sigma N}$ and $\beta=g_{\omega \Lambda} / g_{\omega N}$. Note that the lowest $\chi^{2}$ values are located along a line in this plane (Fig. 4), which was first observed in [13] and

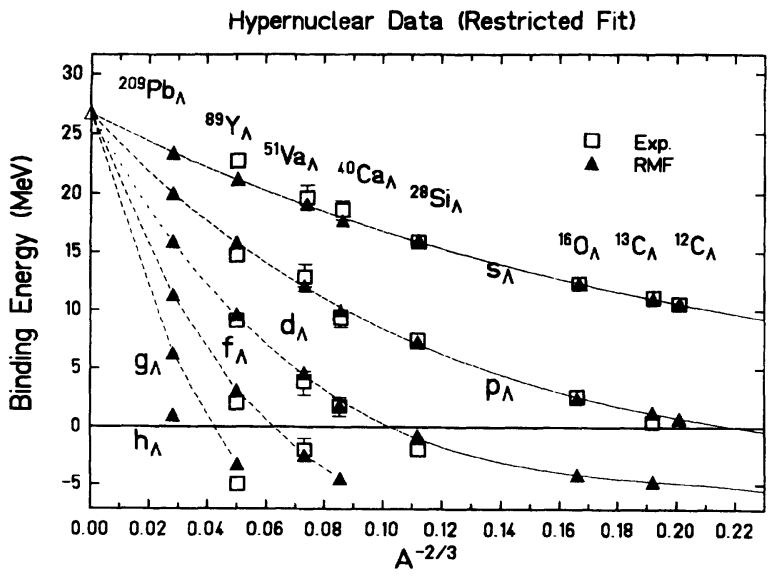

FIG. 2. Single-particle energy of single- $\Lambda$ hypernuclei in the relativistic mean-field theory compared with recent data (restricted fit). 
Light Hypernuclei (Restricted fit)

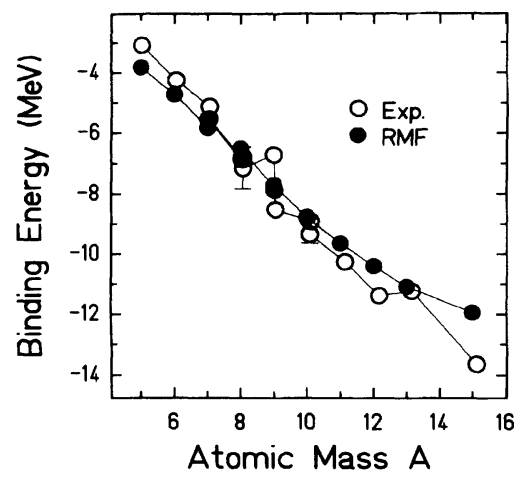

FIG. 3. Binding energy of light hypernuclei in the model compared with experiment.

has recently been confirmed by [34]. This linear dependence is due to the fact that the potential depth of the $\Lambda$ stays constant along this line. The two coupling constants of the $\Lambda$ are connected to the $\Lambda$ potential depth $U_{\Lambda}$ in nuclear matter by the relation

$$
U_{\Lambda}=m_{B}\left(\frac{m_{N}^{*}}{m_{N}}-1\right) \alpha+\frac{g_{\omega}^{2}}{m_{\omega}^{2}} \rho_{0} \beta,
$$

which causes a linear dependence of the constant potential depth on the two parameters $\alpha$ and $\beta$. The effective mass of the $\Lambda$ in nuclear matter,

$$
\frac{m_{\Lambda}^{*}}{m_{\Lambda}}=1-\alpha\left(1-\frac{m_{N}^{*}}{m_{N}}\right),
$$

in the two fit cases is $m_{\Lambda}^{*} / m_{\Lambda}=0.94$ and $m_{\Lambda}^{*} / m_{\Lambda}=0.89$, respectively, which is higher than the value obtained from phenomenological fits to the single- $\Lambda$ particle energies of about $m_{\Lambda}^{*} / m_{\Lambda} \approx 0.80$ [35]. However, even this value can be reproduced within the RMFM by employing a coupling constant $\alpha=0.8$, well within one standard deviation of our best-fit values. Neutron star calculations also indicate a stronger coupling constant for hyperons [36].

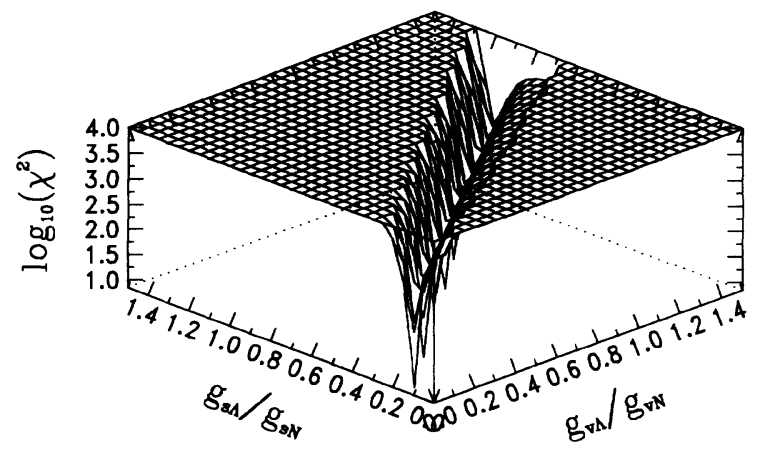

FIG. 4. Squared deviation from the experimental data is plotted vs the scalar and vector coupling constant of the $\Lambda$. The best fit is achieved along a line so that the coupling constants cannot be fixed unambiguously.
Let us now extend the discussion to multi- $\Lambda$ hypernuclei [13].

\section{MULTI- $\Lambda$ HYPERNUCLEI}

In the case of double- $\Lambda$ hypernuclei, one distinguishes between two observables, namely,

$$
\begin{aligned}
& B_{\Lambda \Lambda}\left({ }_{\Lambda \Lambda}^{A} Z\right)=B_{\Lambda}\left({ }_{\Lambda \Lambda}^{A} Z\right)+B_{\Lambda}\left({ }_{\Lambda}^{A-1} Z\right), \\
& \Delta B_{\Lambda \Lambda}\left({ }_{\Lambda \Lambda}^{A} Z\right)=B_{\Lambda}\left({ }_{\Lambda \Lambda}^{A} Z\right)-B_{\Lambda}\left({ }_{\Lambda}^{A-1} Z\right) .
\end{aligned}
$$

The last observable can be used to determine the $\Lambda \Lambda$ interaction. The interaction between hyperons emerges in the RMFM only via the scalar and vector fields; their coupling constants are fixed by the single- $\Lambda$-hypernuclear properties. The model used for the restricted fit reproduces the $B_{\Lambda \Lambda}$ energies (Table II), but the $\Lambda \Lambda$ interaction is evidently too small because of the small coupling. Calculations done for higher coupling constants cannot improve this result. $\Lambda \Lambda$ and $\alpha \alpha$ correlations might be quite important for this very light nuclear systems by increasing $\Delta B_{\Lambda \Lambda}$ (see [37] for calculations including correlations). However, for our discussion of multiple- $\Lambda$ systems, correlations may well be less important. Another hypothetical explanation would be that the double- $\Lambda$ hypernuclei are in fact $H$-dibaryon nuclei $[4,38]$. Here we examine the properties of the spherical double hypernuclei ${ }^{6} \mathrm{He}_{\Lambda \Lambda}$ self-consistently and give a lower limit for the binding of the $\Lambda$ 's. The density distribution (Fig. 5) reveals an interesting feature of multi- $\Lambda$ hypernuclei: The density distribution of the hyperons is shifted outward relative to the nucleons according to the smaller binding. This yields an extended neutral $\Lambda$ halo around the nucleus [13], which results in an enhanced interaction radius. The measured mean lifetime of hypernuclei is approximately $10^{-10} \mathrm{~s}$. These two features are surprisingly similar to the anomaly discussed in [39]. Note that the surface thickness of the $\Lambda$ density is considerably enhanced as compared with that of the nucleons. Therefore the rms radii of the multihypernuclei do not increase drastically; only the tail of the density distribution is enhanced. Cluster model calculations [37] show that correlations push the $\Lambda$ 's farther out (relative to our result). This would also decrease the central density. The strongest relative enhancement of this increase in the interaction radius is found in light hypernuclei [40]. This is due to their shallow binding and the relative higher $\Lambda$ to-nucleon ratio (e.g., for the case ${ }^{6} \mathrm{He}_{\Lambda \Lambda}$, the number of $\Lambda$ 's and neutrons or protons are equal). Experiments

TABLE II. Two observables $\Delta B_{\Lambda \Lambda}$ and $B_{\Lambda \Lambda}$ of the two measured double- $\Lambda$ hypernuclei ${ }^{6} \mathrm{He}_{\Lambda \Lambda}$ and ${ }^{10} \mathrm{Be}_{\Lambda \Lambda}$ as defined in Eq. (2). The theoretical values are compared with the experimental ones [3].

\begin{tabular}{lcccc}
\hline \hline & \multicolumn{2}{c}{$B_{\Lambda \Lambda}(\mathrm{MeV})$} & \multicolumn{2}{c}{$\Delta B_{\Lambda \Lambda}(\mathrm{MeV})$} \\
& Theory & Expt. & Theory & Expt. \\
\hline${ }^{6} \mathrm{He}_{\Lambda \Lambda}$ & 9.0 & $10.9 \pm 0.6$ & 0.6 & $4.7 \pm 0.6$ \\
${ }^{10} \mathrm{Be}_{\Lambda \Lambda}$ & 17.4 & $17.7 \pm 0.4$ & 0.4 & $4.3 \pm 0.4$ \\
\hline \hline
\end{tabular}




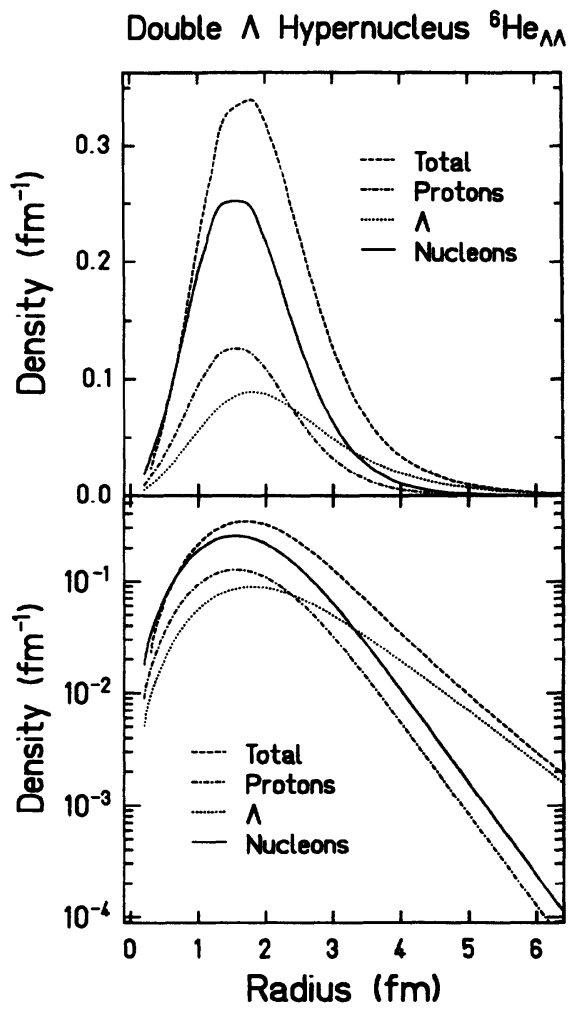

FIG. 5. Density distribution times $r^{2}$ of the double- $\Lambda$ hypernucleus ${ }^{6} \mathrm{He}_{\Lambda \Lambda}$. The $\Lambda$ distribution creates a halo around the nucleons and enhances therefore the interaction radius.

searching for anomalous projectile fragments have excluded almost every charge except for $Z=2$ [39]. Hence the $\Lambda$ corona in light multihypernuclei such as He could be a possible explanation for this hitherto unexplained phenomenon.

Multihypernuclei with more than two hyperons were first discussed in Ref. [12]. The binding energy of different spherical multi- $\Lambda$ hypernuclei is shown in Fig. 6 . They are bound particularly strongly, depending on the

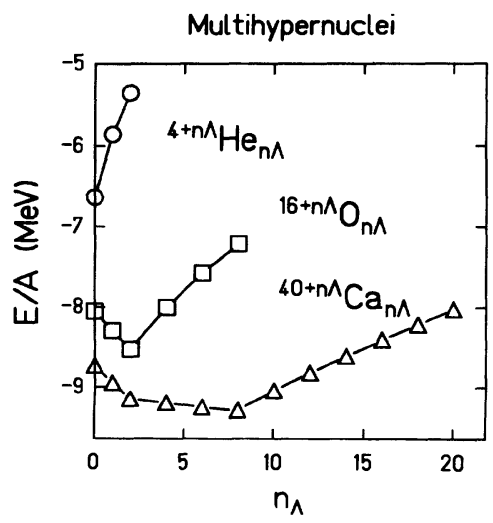

FIG. 6. Binding energy of different multihypernuclei that are dependent on the number of $\Lambda$ 's in the nucleus. Multihypernuclei are more stable than normal nuclei. number of added $\Lambda$ 's. Note that the binding energy increases when hyperons are added to normal nuclei [13]. This is due to the fact that we open a new degree of freedom for which the Fermi energy is small for small numbers of $\Lambda$ 's. The argumentation is analogous to the discussion of the stability of strange quark matter $[16,17,19]$. This is also important for the formation of multihypernuclei in relativistic heavy-ion collisions: The production of multihypernuclei may be favored in the cluster formation phase.

\section{EXOTIC MULTIHYPERNUCLEI}

Now we discuss the structure of even more exotic multihypernuclei. They can be formed by adding two $\Sigma^{-}$to the double hypernuclei ${ }_{\Lambda \Lambda}^{6} \mathrm{He}$. Note that every constituent of this object is in the $1 s_{1 / 2}$ ground state and that the whole "nucleus" has zero charge. Hyperuniversal coupling, i.e., $g_{\Sigma}=g_{\Lambda}$, is suggested from quark models [33]. We adopt this assumption as a first approximation. Bound zero-charge multihypernuclei are indeed found (see Fig. 7) - the central density of these nuclei is by more than $50 \%$ increased as compared with the nucleon density in the ${ }^{4} \mathrm{He}$.

Additional electrically neutral nuclei are found by adding equal numbers of neutrons, protons, $\Lambda$ 's, and $\Sigma$ 's. Figure 7 shows the energy per baryon of such nuclei versus the atomic mass. For atomic masses of $12,36,40$, and 44 , we observe continuum single-particle states; i.e., these objects rapidly evaporate hyperons. All other multistrange nuclei turn out to be stable. Fourfold magic nuclei with closed shells are found for atomic masses of 8 and 32. Even negatively charged nuclei are feasible if more $\Sigma^{-}$'s than protons happen to be present. The mean lifetime of these multistrange objects is short, about $10^{-23} \mathrm{~s}$, because of the strong decay processes

$$
\begin{aligned}
& N+\Sigma \rightarrow N+\Lambda, \\
& \Lambda+\Sigma \rightarrow N+\Xi .
\end{aligned}
$$

The first process is suppressed, as one learns from the narrow width of $\Sigma$-hypernuclei spectra $(\Gamma<5-10 \mathrm{MeV})$

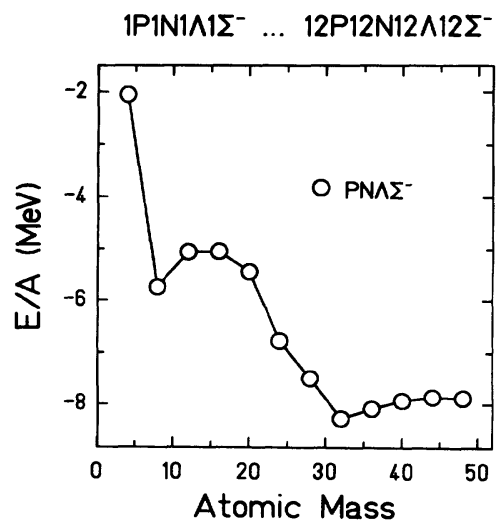

FIG. 7. Binding energy of exotic multihypernuclei consisting of equal numbers of neutrons, protons, $\Lambda$ 's, and $\Sigma$ 's. Note the strong shell effects. 
TABLE III. Configuration of metastable duplets of the nucleons and the hyperons sorted according to strangeness $s$ and charge $q$. The numbers given stand for the sum of the masses (in MeV).

\begin{tabular}{cccccc}
\hline \hline$-s>q$ & -1 & 0 & +1 & +2 \\
\hline 0 & -2 & & $n n$ & $n p$ & $p p$ \\
& & $\Sigma^{-} n$ & 1879 & 1878 & 1877 \\
1 & & $\Lambda n$ & $\Lambda p$ & $\Sigma^{+} n$ \\
& & 2137 & 2055 & 2054 & 2128 \\
2 & $\Sigma^{-} \Sigma^{-}$ & $\Xi^{0} n$ & $\Lambda \Lambda$ & $\Xi^{-} p$ & $\Sigma^{+} \Sigma^{+}$ \\
& 2395 & 2261 & 2231 & 2253 & 2379 \\
3 & $\Xi^{-} \Sigma^{-}$ & $\Xi^{-} \Lambda$ & $\Xi^{0} \Lambda$ & $\Xi^{0} \Sigma^{+}$ & \\
& 2519 & 2437 & 2431 & 2504 & \\
4 & $\Xi^{-} \Xi^{-}$ & $\Xi^{0} \Xi^{-}$ & $\Xi^{0} \Xi^{0}$ & & \\
5 & 2643 & 2636 & 2630 & & \\
5 & $\Xi^{-} \Omega^{-}$ & $\Xi^{0} \Omega^{-}$ & & & \\
& 2994 & 2987 & & & \\
\hline \hline
\end{tabular}

$[8,9]$. The lower mass difference of the second process could yield even stronger suppression. Still they might not live long enough to be experimentally detectable. Are there other metastable configurations of hyperons and nucleons?

\section{METASTABLE EXOTIC MULTIHYPERNUCLEAR OBJECTS: MEMO's}

All possible pairs of nucleons and hyperons can be sorted according to their strangeness number and their charge (both are not changed by strong interactions). The pair with the lowest mass (the most stable) is shown in Table III. Three different baryons can be combined into six possible metastable combinations (e.g., $\Lambda \Xi^{-}$neutron) (see Table IV). It is not possible to have more than three different baryon species in a metastable combination: They will react immediately to form a configuration with two or three different baryons if the mass difference cannot be overcome by binding energy differences inside the bound system.

It is important to note that the combination found (Tables III and IV) cannot decay via meson (pion or kaon) emission into another state. The channels with the smallest $Q$ value are given by the reactions

$$
\begin{aligned}
& \Sigma^{-}+\Sigma^{-} \rightarrow \pi^{+}+\Xi^{-}+n \quad(\Delta E=-6 \mathrm{MeV}), \\
& \Sigma^{+}+\Sigma^{+} \rightarrow \pi^{+}+\Xi^{0}+p \quad(\Delta E=-14 \mathrm{MeV}),
\end{aligned}
$$

where $\Delta E$ denotes the mass difference of the reaction. Binding energy differences in a strange composite can cancel the mass difference, and these strong reactions would be energetically feasible. Therefore metastable combinations which include $\Sigma$ 's might not be bound. Mesonic reaction channels can only occur for the two reactions considered, because all other mesonic channels need a more than $50 \mathrm{MeV}$ binding energy difference in nuclei to overcome the mass difference (i.e., to be exothermic). In view of the maximum binding energy (the potential depth in nuclear matter is $\approx 30 \mathrm{MeV}$ for hyperons, $\approx 50 \mathrm{MeV}$ for nucleons), this seems rather unlikely.

Potential metastable configurations, which might be formed in relativistic heavy-ion collisions, are states consisting of (i) several $\Lambda$ 's, (ii) several $\Lambda^{\prime}$ 's (or $\Sigma^{- \text {'s) with }}$

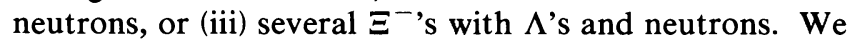
have calculated the total binding energy for a large variety of MEMO's. The critical line for zero binding energy can be compared in the two-dimensional parameter plane to the region of parameter values compatible with hypernuclear data. We find that droplets of lambdas with or without neutrons are less likely to be bound within our model for parameters consistent with hypernuclear data [7]. As mentioned before, the introduction of correlations for the lambdas may change this conclusion because of the magnification of the $\Lambda \Lambda$ interaction.

The double strange hyperon $\Xi$ gives rise to the most in-

TABLE IV. Configuration of metastable triplets of the nucleons and hyperons sorted according to strangeness $s$ and charge $q$.

\begin{tabular}{cccccc}
\hline$\sum_{-s} q$ & -2 & -1 & 0 & +1 & +2 \\
\hline 1 & & & & $\Lambda n p$ & \\
2 & $\Xi^{-} \Sigma^{-} n$ & $\Xi^{-} \Lambda n$ & $\Xi^{0} \Lambda p$ & $\Xi^{0} \Sigma^{+} p$ \\
3 & & $\Xi^{-} \Xi^{0} \Lambda$ & & \\
4 & & & & \\
6 & $\Omega^{-} \Xi^{-} \Xi^{0}$ & & & & \\
7 &
\end{tabular}


triguing speculations: This new degree of freedom can lead to potential effects analogous to those discussed in the context of strangelet stability $[16,17]$ and multihypernuclei (see Fig. 6). As a matter of fact, we cannot fix the coupling of the hyperon $\Xi$ from data. The only data known indicate a potential depth for $\Xi$ 's in nuclear matter of about 20-25 MeV [11]. Therefore we calculate the binding energy of some combinations of neutrons, lambdas, and $\Xi^{-}$'s depending on the two coupling constants of the hyperons (we are choosing, for simplicity, hyperuniversal coupling) and compare these results with the potential depth of the $\Xi$ 's in nuclear matter (Fig. 8). We find certain regions of parameters sets where these objects are bound by several $\mathrm{MeV}$ per baryon and have reasonable potential depths in nuclear matter. Spherical MEMO's, containing two or eight neutrons, $\Lambda$ 's and $\Xi^{- \text {'s }}$ of each, cannot be ruled out. Note that they have a strangeness-to-baryon number fraction of 1 ( $f_{s}=S / A=1$ ), which is in the same range of values as calculated for strange quark matter in its ground state.

The multistrange nuclear systems predicted here [e.g., $\left.2\left(n \Lambda \Xi^{-}\right), 8\left(n \Lambda \Xi^{-}\right)\right]$are negatively charged, while they have a positive baryon number. Recent calculations have shown that a strangelet can also be negatively charged if it is distilled in a ultrarelativistic heavy-ion collision out of a thermally equilibrated quark-gluon plasma [19]. Hence MEMO's seem to have in this respect properties that are quite similar to those of strangelets. Observation of negatively charged nuclei therefore may not be unambiguously related to antinucleus production [41].

The situation changes when, in addition, protons and $\Xi^{0}$ 's are considered. Of all the possible strong reactions between nucleons and all hyperons, only the following two have a mass difference of less than $50 \mathrm{MeV}$ :

$$
\begin{aligned}
& \Xi^{-}+p \rightarrow 2 \Lambda \quad(\Delta E=28 \mathrm{MeV}), \\
& \Xi^{0}+n \rightarrow 2 \Lambda \quad(\Delta E=23 \mathrm{MeV}) .
\end{aligned}
$$

Therefore, if Pauli blocking inhibits these two reactions, we can construct potential MEMO candidates consisting

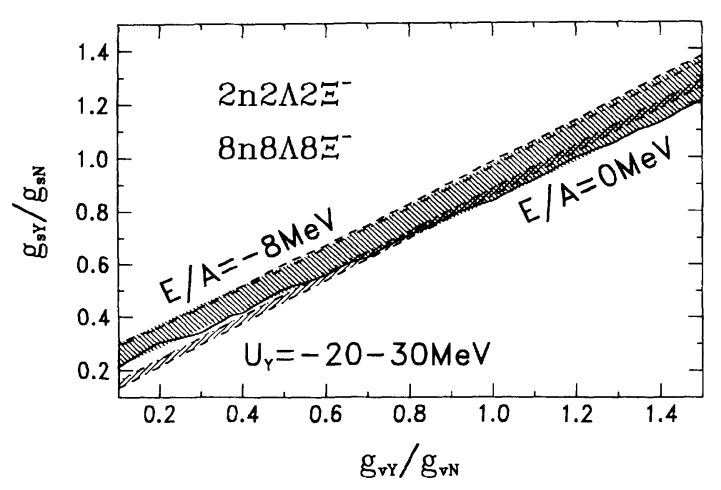

FIG. 8. Binding energy of spherical MEMO's consisting of neutrons, $\Lambda$ 's, and $\Xi^{- \text {'s }}$ is compared with the potential depth of the hyperons in nuclear matter. These negatively charged objects cannot be ruled out at the present stage of knowledge of hypernuclear properties.

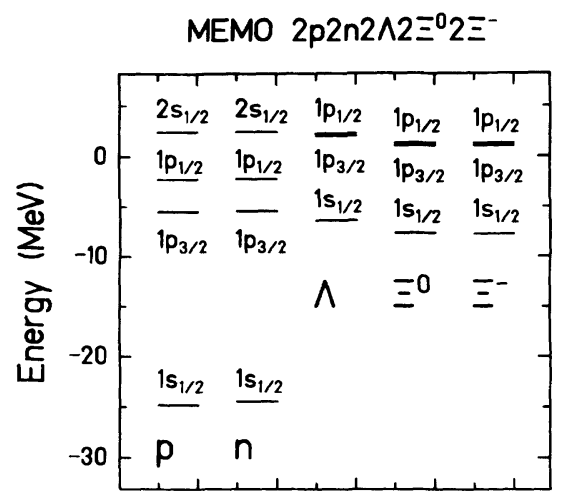

FIG. 9. Single-particle energy of a MEMO consisting of two of each baryon of the baryon octet except the $\Sigma$ 's. The binding energy difference cancels the mass difference of the strong reaction channels so that the whole "nucleus" is metastable.

of $p, n, \Lambda, \Xi^{0}, \Xi^{-}$. The binding energies of the $\Xi$ 's and nucleons must exceed the mass difference of the above two reactions, and all the $\Lambda$ states must be occupied inside the nucleus, in order for Pauli blocking to cause stability. We have indeed been able to construct such a metastable object, namely, a MEMO with two neutrons, protons, $\Lambda$ 's, $\Xi^{-}$'s, and $\Xi^{0}$ 's each (Fig. 9). The sum of the binding energies of the nucleons and $\Xi$ 's cancel the mass difference of the two reaction channels shown above, as can be seen in Fig. 9. Hence these reactions are forbidden energetically, and this exotic object turns out to be metastable. This strange "nucleus" possesses zero charge and a central density $\rho / \rho_{0} \approx 4$, because all baryons reside in their respective $1 s$ state. This is analogous to the enhanced central density $\rho / \rho_{0} \approx 2$ in ${ }^{4} \mathrm{He}$. However, the inclusion of correlations, which will be important for $\alpha$ like objects, shifts the hyperons farther out [37] and gives smaller central densities than obtained with the relativistic mean-field model. Furthermore, the strangeness content of this strange nucleus is $f_{s}=S / A=1$. The formation of such novel, exotic states of nuclear matter in heavy-ion collisions [42] is an intriguing possibility; the production cross section, however, will be very small. We are presently investigating this problem quantitatively based on a covariant coalescence model, using the microscopic baryon distribution from the relativistic quantum molecular-dynamics model as input [43].

\section{SUMMARY AND OUTLOOK}

We have demonstrated that $\Lambda$ hypernuclei can be described rather well in the relativistic mean-field model. Multi- $\Lambda$ hypernuclei are more strongly bound than normal nuclei, which is of great interest for the formation of multihypernuclei in heavy-ion reactions. Exotic multihypernuclei consisting of $\Lambda^{\prime}$ 's and $\Sigma^{-}$'s may be very stable for certain magic numbers. However, their constituents react strongly and these objects are not stable. Metastable exotic multihypernuclear objects (MEMO's) cannot be excluded within the mean-field theory if the coupling 
constants extracted from hypernuclear data are employed. They are negatively charged, but have positive baryon number. Their strangeness number can be equal to their baryon number $\left(f_{s}=1\right)$, similar to the strangelets. If formed, they could be observed easily in relativistic heavy-ion collisions because of their unusual $Z / A$ ratio.

These hadronic objects compete with the strangelets, their quark counterpart. It is, of course, viable that MEMO's form a doorway state to strangelet production in heavy-ion collisions or vice versa. MEMO's may first coalesce in the high multiplicity region of the reaction. If strangelets are more bound than our "conventional" multihypernuclear clusters, the MEMO may then be transformed to a strangelet.

\section{ACKNOWLEDGMENTS}

We like to thank C. B. Dover and P.-G. Reinhard for fruitful discussions. This work was supported by the Gesselschaft für Schwerionenforschung m.b.H. (GSI), the Deutsche Forschungsgemeinschaft (DFG), and the Bundesministerium für Forschung und Technologie (BMFT).
[1] R. E. Chrien and C. B. Dover, Annu. Rev. Nucl. Part. Sci. 39, 113 (1989); H. Bandō, T. Motoba, and J. Žofka, Int. J. Mod. Phys. A 5, 4021 (1990).

[2] M. Jurič et al., Nucl. Phys. B52, 1 (1973).

[3] M. Danysz et al., Nucl. Phys. 49, 121 (1963); Phys. Rev. Lett. 11, 29 (1963); D. J. Browse, ibid. 17, 782 (1966); G. C. Deka, N. R. Saika, and P. Kakati, Indian J. Phys. A 63, 179 (1989).

[4] R. H. Dalitz, D. H. Davis, and A. Deloff, Phys. Lett. B 236, 76 (1990).

[5] S. Aoki et al., Prog. Theor. Phys. 85, 1287 (1991).

[6] C. B. Dover, D. J. Millener, A. Gal, and D. H. Davis, Phys. Rev. C 44, 1905 (1991).

[7] R. E. Chrien, Nucl. Phys. A478, 705c (1988); Nuovo Cimento A 102, 727 (1989).

[8] C. B. Dover, D. J. Millener, and A. Gal, Phys. Rep. 184, 1 (1989).

[9] E. Oset, P. Fernández, L. L. Salcedo, and R. Brockmann, Phys. Rep. 188, 79 (1990).

[10] R. S. Hayano, T. Ishiwaka, M. Iwasaki, H. Outa, E. Takada, H. Tamura, A. Sakaguchi, M. Aoki, and T. Yamazaki, Nuovo Cimento A 102, 437 (1989).

[11] C. B. Dover and A. Gal, Ann. Phys. (N.Y.) 146, 309 (1983).

[12] A. K. Kerman and M. S. Weiss, Phys. Rev. C 8, 408 (1974).

[13] M. Rufa, H. Stöcker, J. Maruhn, P.-G. Reinhard, and W. Greiner, J. Phys. G 13, 143 (1987); J. Mares and J. Žofka, Z. Phys. A 333, 209 (1989); M. Rufa, J. Schaffner, J. A. Maruhn, H. Stöcker, W. Greiner, and P.-G. Reinhard, Phys. Rev. C 42, 2469 (1990); J. Schaffner, C. Greiner, I. N. Mishustin, L. M. Satarov, H. Stöcker, and W. Greiner, in Proceedings of the 19th International Winter Meeting on Nuclear Physics, Bormio, Italy, 1991, edited by I. Iori (unpublished), p. 563; J. Schaffner, C. Greiner, and H. Stöcker, Nucl. Phys. B24B, 246 (1991).

[14] F. Asai, H. Bando, and M. Sano, Phys. Lett. 145B, 19 (1984); M. Wakai, H. Bandō, and M. Sano, Phys. Rev. C 38, 748 (1988); H. Bandō, M. Sano, J. Žofka, and M. Wakai, Nucl. Phys. A501, 900 (1989); H. Bandō, Nuovo Cimento A 102, 627 (1989).

[15] A. U. Abdurakhimov et al., Nuovo Cimento A 102, 645 (1989).

[16] A. R. Bodmer, Phys. Rev. D 4, 1601 (1971).

[17] S. A. Chin and A. K. Kerman, Phys. Rev. Lett. 43, 1292 (1979); E. Witten, Phys. Rev. D 30, 272 (1984); E. Farhi and R. L. Jaffe, ibid. 30, 2379 (1984).

[18] H. Liu and G. L. Shaw, Phys. Rev. D 30, 1137 (1985).

[19] C. Greiner, P. Koch, and H. Stöcker, Phys. Rev. Lett. 58, 1825 (1987); C. Greiner, D. H. Rischke, P. Koch, and H. Stöcker, Phys. Rev. D 38, 2797 (1988); C. Greiner and H. Stöcker, ibid. 44, 3517 (1991).

[20] U. Heinz, K. S. Lee, and M. J. Rhoades-Brown, Mod. Phys. Lett. A 2, 153 (1987); J. Rafelski, Phys. Lett. B 190, 167 (1987); H. W. Barz, B. L. Friman, J. Knoll, and H. Schulz, ibid. 242, 328 (1990); Nucl. Phys. B248, 211 (1991) (see change of conclusions).

[21] B. D. Serot and J. D. Walecka, Adv. Nucl. Phys. 16, 1 (1986).

[22] P.-G. Reinhard, M. Rufa, J. Maruhn, W. Greiner, and J. Friedrich, Z. Phys. A 323, 13 (1986); M. Rufa, J. Maruhn, W. Greiner, P.-G. Reinhard, and M. Strayer, Phys. Rev. C 5, 390 (1988).

[23] P.-G. Reinhard, Rep. Prog. Phys. 52, 439 (1989).

[24] A. Bouyssy, Nucl. Phys. A290, 324 (1977).

[25] R. Brockmann and W. Weise, Phys. Lett. 69B, 167 (1977); Nucl. Phys. A355, 365 (1981).

[26] P.-G. Reinhard, Z. Phys. A 329, 257 (1988).

[27] B. M. Waldhauser, J. A. Maruhn, H. Stöcker, and W. Greiner, Phys. Rev. C 38, 1003 (1987).

[28] J. Boguta and J. R. Bodmer, Nucl. Phys. A292, 414 (1977).

[29] J. Boguta and H. Stöcker, Phys. Lett. 120B, 289 (1983).

[30] A. R. Bodmer and C. E. Price, Nucl. Phys. A405, 123 (1989).

[31] A. R. Bodmer, Nucl. Phys. A526, 703 (1991).

[32] J. Fink, V. Blum, P.-G. Reinhard, J. A. Maruhn, and W. Greiner, Phys. Lett. B 218, 277 (1989).

[33] H. J. Pirner, Phys. Lett. 85B, 190 (1979).

[34] M. Barranco, R. J. Lombard, S. Marcos, and S. A. Moszkowski, Phys. Rev. C 44, 178 (1991).

[35] D. J. Millener, C. B. Dover, and A. Gal, Phys. Rev. C 38, 2700 (1988).

[36] N. K. Glendenning and S. A. Moszkowski, Phys. Rev. Lett. 67, 2414 (1991).

[37] S. Shinmura, Y. Akaishi, and H. Tanaka, Prog. Theor. Phys. 71, 546 (1984); A. R. Bodmer and Q. N. Usmani, Nucl. Phys. A468, 653 (1987).

[38] R. L. Jaffe, Phys. Rev. Lett. 38, 195 (1977).

[39] B. F. Bayman and T. C. Tang, Phys. Rep. 147, 155 (1987).

[40] N. Abou-El-Naga, J. Phys. G 16, 575 (1990).

[41] H. Crawford et al., Search for New Particle and Investigation of Hadronization on Nucleus-Nucleus Collision at the 
AGS, AGS proposal 858, 1990 (unpublished); J. Sandweiss et al., Production of Rare Composite Objects in Relativistic Heavy Ion Collisions, AGS proposal 864, 1990 (unpublished).

[42] C. B. Dover, Nucl. Phys. A527, 635c (1991); Z. Arvey, T.
Csörgö, C. B. Dover, and J. Zimányi (in preparation).

[43] R. Mattiello, J. Schaffner, H. Sorge, H. Stöcker, and C. Greiner, Nucl. Phys. B24B, 221 (1991); J. Aichelin and K. Werner, Phys. Lett. B 274, 260 (1992). 